\title{
THE MONOTONE UNION OF OPEN $n$-CELLS IS AN OPEN $n$-CELL
}

\section{MORTON BROWN}

In a research announcement [2] B. Mazur indicated that modulo the Generalized Schoenflies Theorem, the following theorem could be proved:

"If the open cone over a topological space $X$ is locally Euclidean at the origin, then it is topologically equivalent with Euclidean space."

Ronald Rosen [3] has described an ingenious proof of this theorem based on the now known [1] Generalized Schoenflies Theorem. In the present paper we prove a stronger theorem without employing the Generalized Schoenflies Theorem.

Definitions and notation. If $Q$ is an $n$-cell then $\dot{Q}, \dot{Q}$ denote the interior and boundary of $Q$, respectively. An n-annulus is a homeomorph of $S^{n-1} \times[01]$. If $S$ is an $(n-1)$-sphere in an $n$-cell, then $I(S)$ denotes the interior (complementary domain) of $S$. If $S_{1}, S_{2}$ are $(n-1)$-spheres in an $n$-cell and $S_{1} \subset I\left(S_{2}\right)$, then $\left[S_{1}, S_{2}\right]$ (or equivalently $\left.\left[S_{2}, S_{1}\right]\right)$ denotes the set $\mathrm{Cl}\left[I\left(S_{2}\right)\right]-I\left(S_{1}\right)$. An $(n-1)$-sphere $S$ embedded in a space $X$ is collared if there is a homeomorphism $h$ of $S^{n-1} \times[01]$ into $X$ such that $h\left(S^{n-1} \times 1 / 2\right)=S$. Finally $B_{\tau}$ will denote the $n$-ball of radius $\tau$ in $E^{n}$ and centered at the origin.

LemMa 1. Let $S$ be a collared (n-1)-sphere in the interior of an $n$-cell $Q$ such that $\mathrm{Cl}[I(S)]$ is an $n$-cell. ${ }^{1}$ Let $h$ be a homeomorphism of $Q$ upon itself such that $S \subset I(h(S))$ and $h \mid U=1$ where $U$ is a nonempty open subset of $I(S)$. Then $h(S)$ is a collared $(n-1)$-sphere in $\dot{Q}, \mathrm{Cl}[I(h(S))]$ is an n-cell and $[S, h(S)]$ is an n-annulus.

Proof. Let $f$ be a homeomorphism of $S^{n-1} \times$ [01] into $\dot{Q}$ such that $f\left(S^{n-1} \times 0\right)=S, f\left(S^{n-1} \times[01]\right) \cap h(S)=0$, and $f\left(S^{n-1} \times[01]\right) \cap I(S)=0$. Evidently $I(S) \cup f\left(S^{n-1} \times[01]\right)$ is an $n$-cell. Hence there is a homeomorphism $g$ of $Q$ upon itself such that:

$$
\begin{aligned}
g(S) & \subset U, \\
g f\left(S^{n-1} \times 1 / 2\right) & =S, \\
g \mid h(S) & =1 .
\end{aligned}
$$

Then

Presented to the Society, September 2, 1960; received by the editors September 30, 1960.

1 The results of [1] make this last part of the hypothesis unnecessary. 


$$
\begin{aligned}
g^{-1} h g f\left(S^{n-1} \times[0,1 / 2]\right) & =g^{-1} h g\left[f\left(S^{n-1} \times 0\right), f\left(S^{n-1} \times 1 / 2\right)\right] \\
& =g^{-1} h[g(S), S] \\
& =g^{-1}[g(S), h(S)] \\
& =[S, h(S)] .
\end{aligned}
$$

Hence $[S, h(S)]$ is an $n$-annulus. Obviously $h(S)$ is collared and $\mathrm{Cl}[I(h(S))]$ is an $n$-cell.

LEMMA 2. Let $S$ be a collared (n-1)-sphere in the interior of an $n$-cell $Q$ such that $\mathrm{Cl}[I(S)]$ is an n-cell. ${ }^{1}$ Suppose $M$ is a compact subset of $\dot{Q}$. Then there is a collared $(n-1)$-sphere $S^{\prime}$ in $Q^{\circ}$ such that $I(S) \supset M \cup S$, $\mathrm{Cl}\left[I\left(S^{\prime}\right)\right]$ is an n-cell, and $\left[S, S^{\prime}\right]$ is an n-annulus.

Proof. We may suppose without loss of generality that $Q$ is the unit ball $B_{1}$ in $E^{n}$ and that $I(S)$ contains the origin. Let $\epsilon>0$ be small enough so that $B_{\epsilon} \subset I(S)$ and $M \cup S \subset \dot{B}_{1-\epsilon}$. Let $h$ be a homeomorphism of $B_{1}$ upon itself such that $h \mid B_{\epsilon / 2}=1$ and $h\left(\dot{B}_{\epsilon}\right) \supset B_{1-\epsilon}$. Then $S^{\prime}=h(S)$ contains $M \cup S$ in its interior. Lemma 1 insures that $h(S)$ is collared and that $[S, h(S)]$ is an $n$-annulus.

THEOREM. Let $X$ be a topological space which is the union of a sequence $V_{1} \subset V_{2} \subset \cdots \subset V_{i} \subset \cdots$ of open subsets where each $V_{i}$ is homeomorphic to $E^{n}$. Then $X$ is homeomorphic to $E^{n}$.

PRoof. Let $h_{i}$ map $E^{n}$ homeomorphically onto $V_{i}$. Then $h_{1}\left(B_{1}\right)$ is an $n$-cell in $V_{1}$. There is an integer $n_{2}$ such that

$$
h_{2}\left(\dot{B}_{n_{2}}\right) \supset h_{1}\left(B_{2}\right) \cup h_{2}\left(B_{2}\right) \text {. }
$$

Inductively, there is a sequence of integers $n_{3}, n_{4}, \cdots$, such that for all $i$,

$$
h_{i}\left(\dot{B}_{n_{i}}\right) \supset h_{1}\left(B_{i}\right) \cup \cdots \cup h_{i}\left(B_{i}\right) \cup h_{n_{i-1}}\left(B_{i-1}\right) .
$$

Since $X$ is locally Euclidean, $h_{i}\left(B_{n_{i}}\right)$ is an $n$-cell in $X$ containing $h_{i-1}\left(B_{n_{i-1}}\right)$ in its interior $h_{i}\left(\dot{B}_{n_{i}}\right)$. Finally $\bigcup_{i=1}^{\infty} B_{n_{i}}=X$. For if $x \in X$ there is an integer $j$ such that $x \in V_{j}$. Hence there is an integer $k>j$ such that $x \in h_{j}\left(B_{k}\right)$. But then $x \in h_{k}\left(B_{n_{k}}\right)$. Let $Q_{i}=h_{i}\left(B_{n_{i}}\right)$. Then $X=\cup_{1}^{\infty} Q_{i}$ where $Q_{i}$ is an $n$-cell, $Q_{i} \subset \dot{Q}_{i+1}$, and $\dot{Q}_{i+1}$ is open in $X$.

Let $S_{1}$ be a collared $(n-1)$-sphere in $Q_{1}$ such that $\mathrm{Cl}\left[I\left(S_{1}\right)\right]$ is an $n$-cell. Applying Lemma 2 to the $n$-cell $Q_{2}$, we obtain a collared $(n-1)$ sphere $S_{2}$ in $\grave{Q}_{2}$ such that $I\left(S_{2}\right) \supset Q_{1} \cup S_{1},\left[S_{1}, S_{2}\right]$ is an $n$-annulus, and $\mathrm{Cl}\left[I\left(S_{2}\right)\right]$ is an $n$-cell. The same lemma applied to $Q_{3}$ and $S_{2}$ yields us a collared sphere $S_{3}$ in $Q_{3}$ such that $I\left(S_{3}\right) \supset S_{2} \cup Q_{2},\left[S_{2}, S_{3}\right]$ is an $n$-annulus, and $\mathrm{Cl}\left[I\left(S_{3}\right)\right]$ is an $n$-cell. Continuing this argument, we 
get a sequence $S_{1}, S_{2}, \cdots$, of $(n-1)$-spheres such that $\left[S_{i}, S_{i+1}\right]$ is an $n$-annulus and $X=I\left(S_{1}\right) \cup\left[S_{1}, S_{2}\right] \cup\left[S_{3}, S_{4}\right] \cup \ldots$ Evidently $X$ is homeomorphic to $E^{n}$.

\section{REFERENCES}

1. Morton Brown, A proof of the generalized Schoenfies theorem, Bull. Amer. Math. Soc. vol. 66 (1960) pp. 74-76. p. 65 .

2. Barry Mazur, Embeddings of spheres, Bull. Amer. Math. Soc. vol. 65 (1959)

3. Ronald Rosen, $A$ weak form of the star conjecture for manifolds, Abstract 570-28, Notices Amer. Math. Soc. vol. 7 (1960) p. 380.

UNIVERSITY OF MichigAN

\section{THREADS WITHOUT IDEMPOTENTS}

\section{R. STOREY 1}

If a thread $S$ has no idempotents and if $S^{2}=S$, then $S$ is iseomorphic with the real interval $(0,1)$ under ordinary multiplication $[2$, Corollary 5.6]. Although the result is not nearly as pleasing as the special case just quoted, we shall give here a description of any thread without idempotents. Recall from [1] that a thread is a connected topological semigroup in which the topology is that induced by a total order.

First some examples. Let $X$ be a totally ordered set which is a connected space in the interval topology, let $T$ be a subset of $X$ containing, with $t$, all elements less than $t$, and let $\phi$ be any continuous function from $X$ into $(0,1)$ whose restriction, $\phi_{0}$, to $T$ is a strictly order-preserving map of $T$ onto $\left(0, a^{2}\right)$ where $a=1$.u.b. $\phi(X)$. (We admit that $a$ might be 1.) For such a $\phi$ to exist it is evidently necessary that $X$ not have a least element, that $T$ not have a greatest element and, provided $T \neq X$ so that the least upper bound, $q$, of $T$ exists, that $\phi(q)=a^{2}$.

If $\phi(X)$ is the open interval $(0, a)$, define a multiplication in $X$ by: $x \circ y=\phi_{0}^{-1}(\phi(x) \phi(y))$. With this definition it is quite easy to see that $X$ is a thread without idempotents and that $\phi$ is a homomorphism.

In the event that $\phi(X)$ is the half closed interval $(0, a]$ (which implies of course that $a<1)$, put $A=\phi^{-1}(a)$ and $B=\phi^{-1}\left(a^{2}\right)$, observe that $q$ must be the least element of $B$, and let $\psi$ be any continuous

Received by the editors July 28, 1960.

1 This paper was prepared while the author held a National Science Foundation Postdoctoral Fellowship. 